\title{
RAYLEIGH WAVES IN A VISCOELASTIC HALF-SPACE UNDER INITIAL HYDROSTATIC STRESS IN PRESENCE OF THE TEMPERATURE FIELD
}

\author{
SUSHIL KUMAR ADDY AND NIL RATAN CHAKRABORTY \\ Received 1 February 2005 and in revised form 28 October 2005
}

The effect of the temperature and initial hydrostatic stress has been shown on the propagation of Rayleigh waves in a viscoelastic half-space. It has been explained how the velocity of Rayleigh waves depends not only on the parameters pertaining to the viscoelastic properties of the half-space, but on the temperature and the initial hydrostatic stress of the half-space also. The variations of the phase velocity of Rayleigh waves in dimensionless form with respect to the magnitude of the initial hydrostatic stress under certain practical assumptions have been depicted in graphs after numerical computations. If the temperature and the initial hydrostatic stress of the half-space are neglected, the results obtained are in perfect agreement with the classical case as obtained by Caloi for the propagation of Rayleigh waves in a viscoelastic medium.

\section{Introduction}

The propagation of thermoelastic waves has been discussed long ago by Lockett [5] and Nowacki and Sokołowski [7] in different media. Recently, this has been explained in a different manner by some authors such as Chandrasekharaiah [3]. The effect of viscosity on the propagation of these waves has also been shown by a few authors such as Das and Sengupta [4]. But none of them considered the initial stress that might be present in the media. But the earth is an initially stressed medium. Hence it should be of geophysical interest to see how the initial stress influences the propagation of waves in elastic or a viscoelastic medium when the medium is heated.

This paper has discussed the effect of the temperature as well as the initial hydrostatic stress on the propagation of Rayleigh waves in a viscoelastic half-space. Here, a new frequency equation of viscoelastic Rayleigh waves has been derived, which involves the parameters connected with the temperature and the initial hydrostatic stress besides the viscoelastic properties of the half-space. The values of the phase velocity of Rayleigh waves have been computed for different values of the initial hydrostatic stress of the halfspace in dimensionless form for certain values of the coupling coefficient of temperature and strain fields. These graphs show that the phase velocity of Rayleigh waves changes 


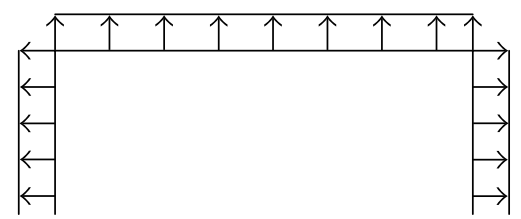

Figure 2.1. Viscoelastic half-space under initial hydrostatic stress in presence of temperature field.

remarkably with respect to the initial hydrostatic stress of the half-space as well as the coupling coefficient of temperature and strain fields of the medium.

\section{Formulation and solution of the problem}

Let us consider a Voigt-type viscoelastic half-space $y \geq 0$, the boundary of which $y=0$ is free from tractions, but does permit heat exchange with the surroundings. Let the halfspace be under an initial hydrostatic stress $H$ at an initial temperature $T_{o}$ (see Figure 2.1). When the temperature of the half-space is changed, incremental thermal stresses $s_{i j}$ together with incremental strains $e_{i j}$ are produced in it, which are measured with reference to the rotated axes as explained by [1].

The dynamical equations of equilibrium under initial hydrostatic stress are given by [1]

$$
\frac{\partial s_{11}}{\partial x}+\frac{\partial s_{12}}{\partial y}=\rho \frac{\partial^{2} u}{\partial t^{2}}, \quad \frac{\partial s_{12}}{\partial x}+\frac{\partial s_{22}}{\partial y}=\rho \frac{\partial^{2} v}{\partial t^{2}} .
$$

Here, $s_{11}, s_{22}$ are the incremental normal thermal stresses along $x$ - and $y$-axes, respectively. $s_{12}$ is the incremental shear thermal stress in the $x y$ plane. $u$ and $v$ are the displacement components along $x$ - and $y$-axes, respectively.

The stress-strain relations in the Voigt-type viscoelastic half-space under thermal condition are given by

$$
\begin{gathered}
s_{11}=\left[(\lambda+2 \mu)+\left(\lambda^{\prime}+2 \mu^{\prime}\right) \frac{\partial}{\partial t}\right] e_{x x}+\left[\lambda+\lambda^{\prime} \frac{\partial}{\partial t}\right] e_{y y}-\gamma T, \\
s_{22}=\left[\lambda+\lambda^{\prime} \frac{\partial}{\partial t}\right] e_{x x}+\left[(\lambda+2 \mu)+\left(\lambda^{\prime}+2 \mu^{\prime}\right) \frac{\partial}{\partial t}\right] e_{y y}-\gamma T, \\
s_{12}=2\left[\mu+\mu^{\prime} \frac{\partial}{\partial t}\right] e_{x y},
\end{gathered}
$$

where $\gamma=(3 \lambda+2 \mu) \alpha_{t}, \alpha_{t}$ is the coefficient of linear expansion, and $T$ is the incremental change of temperature from the initial state. The incremental strain components are given by [6]

$$
e_{x x}=\frac{\partial u}{\partial x}, \quad e_{y y}=\frac{\partial v}{\partial y}, \quad e_{x y}=\frac{1}{2}\left[\frac{\partial v}{\partial x}+\frac{\partial u}{\partial y}\right]
$$




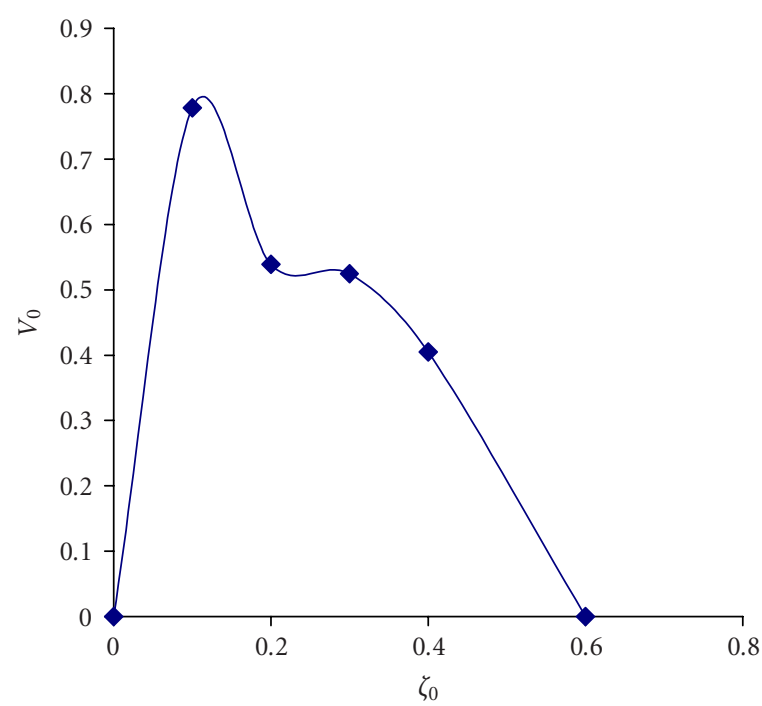

Figure 2.2. $\in=-0.1$.

Equation (2.1) with the help of (2.2) and (2.3) changes to

$$
\begin{gathered}
{\left[\lambda+2 \mu+\left(\lambda^{\prime}+2 \mu^{\prime}\right) \frac{\partial}{\partial t}\right] \frac{\partial^{2} u}{\partial x^{2}}+\left[(\lambda+\mu)+\left(\lambda^{\prime}+\mu^{\prime}\right) \frac{\partial}{\partial t}\right] \frac{\partial^{2} v}{\partial x \partial y}} \\
+\left[\mu+\mu^{\prime} \frac{\partial}{\partial t}\right] \frac{\partial^{2} u}{\partial y^{2}}=\rho \frac{\partial^{2} u}{\partial t^{2}}+\gamma \frac{\partial T}{\partial x}, \\
{\left[\lambda+2 \mu+\left(\lambda^{\prime}+2 \mu^{\prime}\right) \frac{\partial}{\partial t}\right] \frac{\partial^{2} v}{\partial y^{2}}+\left[\lambda+\mu+\left(\lambda^{\prime}+\mu^{\prime}\right) \frac{\partial}{\partial t}\right] \frac{\partial^{2} u}{\partial x \partial y}} \\
+\left(\mu+\mu^{\prime} \frac{\partial}{\partial t}\right) \frac{\partial^{2} v}{\partial x^{2}}=\rho \frac{\partial^{2} v}{\partial t^{2}}+\gamma \frac{\partial T}{\partial y} .
\end{gathered}
$$

The displacement components $u$ and $v$ may be expressed in terms of the potential functions $\phi$ and $\psi$ as follows:

$$
u=\frac{\partial \phi}{\partial x}-\frac{\partial \psi}{\partial y}, \quad v=\frac{\partial \phi}{\partial y}+\frac{\partial \psi}{\partial x} .
$$

Equations (2.4) and (2.5) show that potential functions $\phi$ and $\psi$ satisfy the wave equations

$$
\begin{gathered}
(\lambda+2 \mu) \nabla^{2} \phi+\left(\lambda^{\prime}+2 \mu^{\prime}\right) \frac{\partial}{\partial t}\left(\nabla^{2} \phi\right)=\rho \frac{\partial^{2} \phi}{\partial t^{2}}+\gamma T, \\
\mu \nabla^{2} \psi+\mu^{\prime} \frac{\partial}{\partial t}\left(\nabla^{2} \psi\right)=\rho \frac{\partial^{2} \psi}{\partial t^{2}},
\end{gathered}
$$

where $\nabla^{2}=\partial^{2} / \partial x^{2}+\partial^{2} / \partial y^{2}$. 


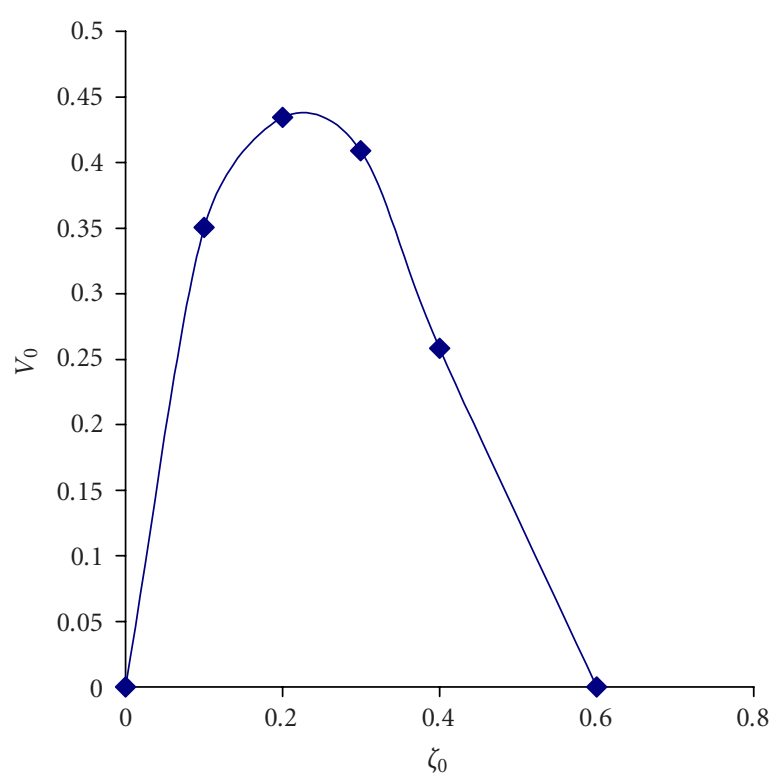

Figure 2.3. $\in=0$.

The heat conduction equation is given by [6]

$$
\nabla^{2} T-\frac{S \rho}{\delta} \frac{\partial T}{\partial t}-\frac{\gamma T_{o}}{\delta} \nabla^{2} \frac{\partial \phi}{\partial t}=0
$$

where $S$ is the specific heat capacity and $\delta$ is the thermal conductivity of the medium.

To find the solution of (2.6a), (2.6b), and (2.7), it is assumed that

$$
\begin{aligned}
& \phi(x, y, t)=\phi_{1}(y) \exp [i(\kappa x-\omega t)], \\
& \psi(x, y, t)=\psi_{1}(y) \exp [i(\kappa x-\omega t)], \\
& T(x, y, t)=T_{1}(y) \exp [i(\kappa x-\omega t)],
\end{aligned}
$$

which are plane harmonic waves moving along the $x$-axis.

Using (2.8a) and (2.8c), $T$ is eliminated from (2.6a) and (2.7), and we get

$$
\left[\nabla^{2}-\frac{S \rho}{\delta} \frac{\partial}{\partial t}\right]\left[\nabla^{2}-\frac{1}{c_{1}^{2}-i \omega c_{1}^{\prime 2}} \frac{\partial^{2}}{\partial t^{2}}\right] \phi-\frac{\gamma^{2} T_{o}}{\left[(\lambda+2 \mu)-i \omega\left(\lambda^{\prime}+2 \mu^{\prime}\right)\right] \delta} \nabla^{2}\left(\frac{\partial \phi}{\partial t}\right)=0
$$

Equation (2.6b) with the help of (2.8b) can be written as

$$
\left[\nabla^{2}-\frac{1}{c_{2}^{2}-i \omega c_{2}^{\prime 2}} \frac{\partial^{2}}{\partial t^{2}}\right] \psi=0
$$


where

$$
c_{1}^{2}=\frac{(\lambda+2 \mu)}{\rho}, \quad c_{1}^{\prime 2}=\frac{\left(\lambda^{\prime}+2 \mu^{\prime}\right)}{\rho}, \quad c_{2}^{2}=\frac{\mu}{\rho}, \quad c_{2}^{\prime 2}=\frac{\mu^{\prime}}{\rho} .
$$

Introducing (2.8a) into (2.9a) and (2.8b) into (2.9b), we obtain the following differential equations:

$$
\begin{gathered}
\left(\frac{\partial^{2}}{\partial y^{2}}-\lambda_{1}^{2}\right)\left(\frac{\partial^{2}}{\partial y^{2}}-\lambda_{2}^{2}\right) \phi_{1}(y)=0, \\
\left(\frac{\partial^{2}}{\partial y^{2}}-\nu^{2}\right) \psi_{1}(y)=0,
\end{gathered}
$$

where

$$
\lambda_{1}^{2}=\kappa^{2}-k_{1}^{2}, \quad \lambda_{2}^{2}=\kappa^{2}-k_{2}^{2}, \quad \nu^{2}=\kappa^{2}-\tau^{2}, \quad \tau=\frac{\omega^{2}}{\left(c_{2}^{2}-i \omega c_{2}^{\prime 2}\right)} .
$$

Here, $k_{1}^{2}$ and $k_{2}^{2}$ are the roots of the biquadratic equation

$$
k^{4}-k^{2}\left[\sigma^{2}+q(1+\in)\right]+q \sigma^{2}=0,
$$

where $k^{2}=-\nabla^{2}$ and the roots $k_{1}^{2}$ and $k_{2}^{2}$ are given by

$$
k_{1}^{2}=q\left[1+\frac{q \in}{q-\sigma^{2}}\right], \quad k_{2}^{2}=\sigma^{2}\left[1-\frac{q \in}{q-\sigma^{2}}\right],
$$

where

$$
\sigma^{2}=\frac{\omega^{2}}{c_{1}^{2}-i \omega c_{1}^{\prime 2}}, \quad q=\frac{i \omega S \rho}{\delta}, \quad \in=\frac{\gamma^{2} T_{o}}{S \rho\left[(\lambda+2 \mu)-i \omega\left(\lambda^{\prime}+2 \mu^{\prime}\right)\right]} .
$$

The requirement that the stresses and hence the potential functions $\phi$ and $\psi$ vanish as $x^{2}+y^{2}$ tends to infinity leads to the following solution of (2.11):

$$
\begin{gathered}
\phi_{1}=A e^{-\lambda_{1} y}+B e^{-\lambda_{2} y}, \\
\psi_{1}=C e^{-v y} .
\end{gathered}
$$

Combining (2.8a), (2.8b), and (2.16a), (2.16b) respectively, we get

$$
\begin{gathered}
\phi(x, y, t)=\left\lfloor A e^{-\lambda_{1} y}+B e^{-\lambda_{2} y}\right\rfloor \exp [i(\kappa x-\omega t)], \\
\psi(x, y, t)=C e^{-v y} \exp [i(\kappa x-\omega t)] .
\end{gathered}
$$

Using (2.6a), (2.8c), and (2.17a), we get

$$
T=\frac{\rho m^{2}}{\gamma}\left[A \eta_{1} e^{-\lambda_{1} y}+B \eta_{2} e^{-\lambda_{2} y}\right] \exp [i(\kappa x-\omega t)]
$$




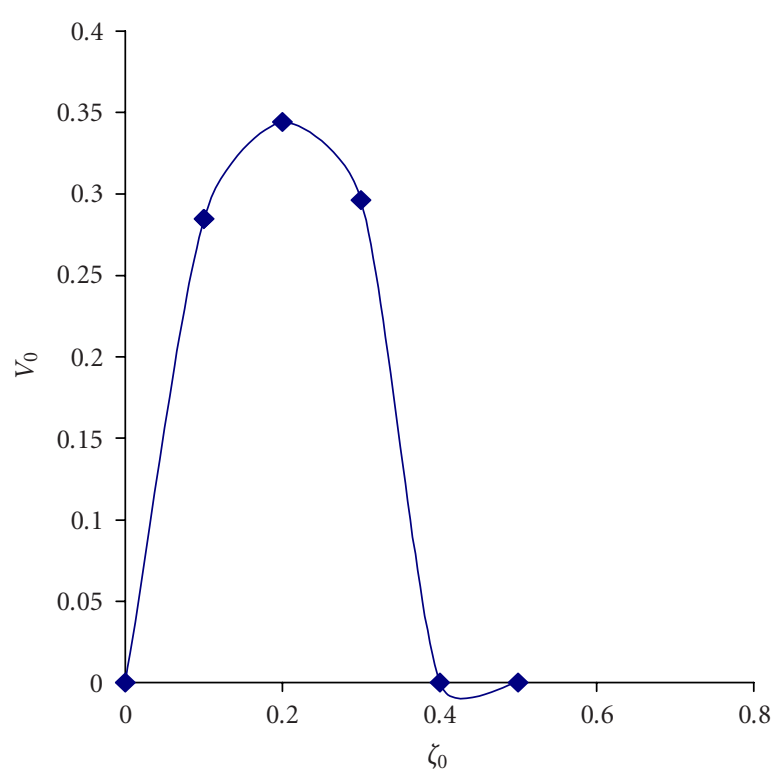

Figure 2.4. $\in=+0.1$. Variation of velocity of Rayleigh waves with initial stress in viscoelastic medium when $\lambda=0$ and $\mu=0$ (Figures 2.2, 2.3, 2.4).

where

$$
m^{2}=c_{1}^{2}-i \omega c_{1}^{\prime 2}, \quad \eta_{1}=\sigma^{2}-k_{1}^{2}, \quad \eta_{2}=\sigma^{2}-k_{2}^{2}
$$

The boundary conditions on the plane $y=0$ are given by [1]

$$
\Delta f_{x}=s_{12}-H \frac{\partial v}{\partial x}=0, \quad \Delta f_{y}=s_{22}+H \frac{\partial u}{\partial x}=0, \quad \frac{\partial T}{\partial y}+h T=0 .
$$

Here, $\Delta f_{x}$ and $\Delta f_{y}$ are incremental boundary forces per unit initial area along $x$ - and $y$-axes, respectively, and $h$ is the ratio between the coefficient of heat transfer and thermal conductivity.

By using (2.2), (2.3), (2.5), (2.17a), (2.17b), and (2.20), we change these boundary conditions to

$$
\begin{gathered}
A\left[\kappa \lambda_{1}\left\{i(2 \mu-H)+2 \omega \mu^{\prime}\right\}\right]+B\left[\kappa \lambda_{2}\left\{i(2 \mu-H)+2 \omega \mu^{\prime}\right\}\right] \\
+C\left[\kappa^{2}\left\{(2 \mu-H)-2 i \omega \mu^{\prime}\right\}-\tau^{2}\left(\mu-i \omega \mu^{\prime}\right)\right]=0, \\
A\left[\rho m^{2}\left(\kappa^{2}-\sigma^{2}\right)-\kappa^{2}\left\{(\lambda+H)-i \omega \lambda^{\prime}\right\}\right]+B\left[\rho m^{2}\left(\kappa^{2}-\sigma^{2}\right)-\kappa^{2}\left\{(\lambda+H)-i \omega \lambda^{\prime}\right\}\right] \\
+C\left[-\kappa \nu\left\{2 \mu^{\prime} \omega+i(2 \mu-H)\right\}\right]=0, \\
A\left[\eta_{1}\left(h-\lambda_{1}\right)\right]+B\left[\eta_{2}\left(h-\lambda_{2}\right)\right]=0 .
\end{gathered}
$$


Eliminating $A, B, C$ from (2.21), we get the following frequency equation of Rayleigh waves:

$$
\left[\begin{array}{ccc}
{\left[\kappa \lambda_{1}\left\{i(2 \mu-H)+2 \mu^{\prime} \omega\right\}\right]} & {\left[\kappa \lambda_{2}\left\{i(2 \mu-H)+2 \mu^{\prime} \omega\right\}\right]} & {\left[\kappa^{2}\left\{(2 \mu-H)-2 i \omega \mu^{\prime}\right\}\right.} \\
{\left[\rho m^{2}\left(\kappa^{2}-\sigma^{2}\right)\right.} & \left.-\tau^{2}\left(\mu-i \omega \mu^{\prime}\right)\right] \\
\left.-\kappa^{2}\left(\lambda+H-i \omega \lambda^{\prime}\right)\right] & \left.-\kappa^{2}\left(\lambda+H-i \omega \lambda^{\prime}\right)\right] & {\left[-\kappa \nu\left\{2 \mu^{\prime} \omega+i(2 \mu-H)\right\}\right]} \\
\eta_{1}\left(h-\lambda_{1}\right) & \eta_{2}\left(h-\lambda_{2}\right) & 0
\end{array}\right]=0 .
$$

If we ignore the presence of the initial stress and the temperature field in the half-space, then $H$ and $q$ both vanish, $h=0$ and $\lambda_{1}=\kappa$ and (2.22) reduces to

$$
\left[\begin{array}{ccc}
{\left[\kappa^{2} \frac{2 i \rho \omega^{2}}{k_{\beta}^{2}}\right]} & {\left[\kappa \sqrt{\kappa^{2}-k_{\alpha}^{2}} \frac{2 i \rho \omega^{2}}{k_{\beta}^{2}}\right]} & {\left[\frac{2 \kappa^{2} \rho \omega^{2}}{k_{\beta}^{2}}-\rho \omega^{2}\right]} \\
{\left[\frac{\rho \omega^{2}}{k_{\alpha}^{2}}\left(\kappa^{2}-k_{\alpha}^{2}\right)-\kappa^{2} \rho \omega^{2}\left(\frac{1}{k_{\alpha}^{2}}-\frac{2}{k_{\beta}^{2}}\right)\right]} & {\left[\frac{\rho \omega^{2}}{k_{\alpha}^{2}}\left(\kappa^{2}-k_{\alpha}^{2}\right)-\kappa^{2} \rho \omega^{2}\left(\frac{1}{k_{\alpha}^{2}}-\frac{2}{k_{\beta}^{2}}\right)\right]} & {\left[\begin{array}{cc}
-\frac{2 i \rho \omega^{2}}{k_{\beta}^{2}} \kappa \sqrt{\kappa^{2}-k_{\beta}^{2}} \\
-\kappa k_{\alpha}^{2}
\end{array}\right.} \\
0 & 0
\end{array}\right]=0,
$$

where we have used

$$
k_{\alpha}^{2}=\frac{\rho \omega^{2}}{(\lambda+2 \mu)-i \omega\left(\lambda^{\prime}+2 \mu^{\prime}\right)}, \quad k_{\beta}^{2}=\frac{\rho \omega^{2}}{\mu-i \omega \mu^{\prime}} .
$$

Expanding the determinant (2.23) and simplifying, we get

$$
1-8 \frac{\kappa^{2}}{k_{\beta}^{2}}+\left[24-16 \frac{k_{\alpha}^{2}}{k_{\beta}^{2}}\right] \frac{\kappa^{4}}{k_{\beta}^{4}}-16\left(1-\frac{k_{\alpha}^{2}}{k_{\beta}^{2}}\right) \frac{\kappa^{6}}{k_{\beta}^{6}}=0,
$$

which is the same equation as obtained by Caloi [2] for the propagation of Rayleigh waves in a viscoelastic half-space without initial stress and temperature.

But considering the initial hydrostatic stress and the temperature field to be present in the half-space and assuming that $\lambda=\mu$ and $\lambda^{\prime}=(2 / 3) \mu^{\prime} ; \sigma, k_{1}, k_{2}$, and $\in$ all change, respectively, to $\sigma^{\prime}, k_{1}^{\prime}, k_{2}^{\prime}$, and $\epsilon^{\prime}$ are given by

$$
\begin{gathered}
\sigma^{\prime 2}=\frac{\omega^{2}}{c_{01}^{2}-i \omega c_{01}^{\prime 2}}, \quad c_{01}^{2}=\frac{2 \mu}{\rho}, c_{01}^{\prime 2}=\frac{8 \mu^{\prime}}{3 \rho} \\
k_{1}^{\prime 2}=q\left[1+\frac{q \epsilon^{\prime}}{q-\sigma^{\prime 2}}\right], \quad k_{2}^{\prime 2}=\sigma^{\prime 2}\left[1-\frac{q \epsilon^{\prime}}{q-\sigma^{\prime 2}}\right], \quad \in^{\prime}=\frac{3 \gamma^{2} T_{o}}{S \rho\left[9 \mu-i \omega 8 \mu^{\prime}\right]} .
\end{gathered}
$$

Then, (2.22) reduces to

$$
\left[\begin{array}{ccc}
2 i \beta_{1} \frac{\left(x \mu-i \omega \mu^{\prime}\right)}{\left(\mu-i \omega \mu^{\prime}\right)} & 2 i \beta_{2} \frac{\left(x \mu-i \omega \mu^{\prime}\right)}{\left(\mu-i \omega \mu^{\prime}\right)} & \frac{\tau^{2}}{\kappa^{2}}-\frac{2\left(x \mu-i \omega \mu^{\prime}\right)}{\left(\mu-i \omega \mu^{\prime}\right)} \\
\frac{2\left(x \mu-i \omega \mu^{\prime}\right)}{\left(\mu-i \omega \mu^{\prime}\right)}-\frac{\tau^{2}}{\kappa^{2}} & \frac{2\left(x \mu-i \omega \mu^{\prime}\right)}{\left(\mu-i \omega \mu^{\prime}\right)}-\frac{\tau^{2}}{\kappa^{2}} & \frac{2 i \beta_{3}\left(x \mu-i \omega \mu^{\prime}\right)}{\left(\mu-i \omega \mu^{\prime}\right)} \\
\eta_{1}\left(h-\kappa \beta_{1}\right) & \eta_{2}\left(h-\kappa \beta_{2}\right) & 0
\end{array}\right]=0
$$


where

$$
\beta_{1}=\sqrt{1-\frac{k_{1}^{\prime 2}}{\kappa^{2}}}, \quad \beta_{2}=\sqrt{1-\frac{k_{2}^{\prime 2}}{\kappa^{2}}}, \quad \beta_{3}=\sqrt{1-\frac{\tau^{2}}{\kappa^{2}}}, \quad x=1-\frac{H}{2 \mu} .
$$

Expanding the determinant in (2.27), we get

$$
\left[\frac{2\left(x \mu-i \omega \mu^{\prime}\right)}{\left(\mu-i \omega \mu^{\prime}\right)}-\frac{\tau^{2}}{\kappa^{2}}\right]^{2}=4 \beta_{3}\left[\frac{x \mu-i \omega \mu^{\prime}}{\mu-i \omega \mu^{\prime}}\right]^{2}\left[\frac{\kappa \beta_{1} \beta_{2}\left(\eta_{1}-\eta_{2}\right)+h\left(\beta_{1} \eta_{2}-\beta_{2} \eta_{1}\right)}{h\left(\eta_{2}-\eta_{1}\right)+\kappa\left(\beta_{1} \eta_{1}-\beta_{2} \eta_{2}\right)}\right] .
$$

In deducing this equation, we assumed a convection condition for the temperature on the boundary of the half-space. For thermal insulation, $h=0$ and (2.29) reduces to

$$
\left[\frac{2\left(x \mu-i \omega \mu^{\prime}\right)}{\left(\mu-i \omega \mu^{\prime}\right)}-\frac{\tau^{2}}{\kappa^{2}}\right]^{2}=4 \beta_{3}\left[\frac{x \mu-i \omega \mu^{\prime}}{\mu-i \omega \mu^{\prime}}\right]^{2} \frac{\beta_{1} \beta_{2}\left(\eta_{1}-\eta_{2}\right)}{\left(\beta_{1} \eta_{1}-\beta_{2} \eta_{2}\right)} .
$$

Assuming heat transfer to be infinitely large, $h=\infty$ and (2.29) reduces to

$$
\left[\frac{2\left(x \mu-i \omega \mu^{\prime}\right)}{\left(\mu-i \omega \mu^{\prime}\right)}-\frac{\tau^{2}}{\kappa^{2}}\right]^{2}=4 \beta_{3}\left[\frac{x \mu-i \omega \mu^{\prime}}{\mu-i \omega \mu^{\prime}}\right]^{2} \frac{\left(\beta_{1} \eta_{2}-\beta_{2} \eta_{1}\right)}{\left(\eta_{2}-\eta_{1}\right)} .
$$

Expressing the quantities $\lambda_{1}, \lambda_{2}, \nu, \eta_{1}$, and $\eta_{2}$ in terms of the quantities $\beta_{1}, \beta_{2}$, and $\beta_{3}$, we find that (2.29) reduces to

$$
\begin{aligned}
& {\left[\frac{2\left(x \mu-i \omega \mu^{\prime}\right)}{\left(\mu-i \omega \mu^{\prime}\right)}-\frac{c^{2}}{c_{2}^{2}-i \omega c_{2}^{\prime 2}}\right]^{2}\left[\beta_{1}^{2}+\beta_{2}^{2}+\beta_{1} \beta_{2}-1+\frac{c^{2}}{\left(c_{01}^{2}-i \omega c_{01}^{\prime 2}\right)}\right]} \\
& -4\left[\frac{x \mu-i \omega \mu^{\prime}}{\mu-i \omega \mu^{\prime}}\right]^{2} \beta_{1} \beta_{2} \beta_{3}\left(\beta_{1}+\beta_{2}\right) \\
& =\frac{h}{\kappa}\left[\left\{\frac{2\left(x \mu-i \omega \mu^{\prime}\right)}{\left(\mu-i \omega \mu^{\prime}\right)}-\frac{c^{2}}{c_{2}^{2}-i \omega c_{2}^{\prime 2}}\right\}\left(\beta_{1}+\beta_{2}\right)\right. \\
& \left.\quad-4\left(\frac{x \mu-i \omega \mu^{\prime}}{\mu-i \omega \mu^{\prime}}\right)^{2} \beta_{3}\left(\beta_{1} \beta_{2}+1-\frac{c^{2}}{c_{01}^{2}-i \omega c_{01}^{\prime 2}}\right)\right]
\end{aligned}
$$

where $c^{2}=\omega^{2} / \kappa^{2}$ is the phase velocity of Rayleigh waves.

The quantity $1 / \operatorname{Re}(1 / c)$ is a measure of the phase velocity and $\omega I_{m}(1 / c)$ is a measure of the damping of Rayleigh waves propagating along the positive $x$-axis.

Under the assumptions $\lambda=\mu$ and $\lambda^{\prime}=(2 / 3) \mu^{\prime},(2.13)$ changes to

$$
k_{1}^{\prime 2}+k_{2}^{\prime 2}=\sigma^{\prime 2}+q\left(1+\epsilon^{\prime}\right), \quad k_{1}^{\prime 2} k_{2}^{\prime 2}=\sigma^{\prime 2} q .
$$


Using (2.28) and (2.33), we get

$$
\begin{gathered}
\beta_{1}^{2}+\beta_{2}^{2}=2-\frac{c^{2}}{c_{01}^{2}-i \omega c_{01}^{\prime 2}}-\frac{i c^{2}\left(1+\epsilon^{\prime}\right)}{f\left(c_{01}^{2}-i \omega c_{01}^{\prime 2}\right)} \\
\beta_{1}^{2} \beta_{2}^{2}=1-\frac{c^{2}}{c_{01}^{2}-i \omega c_{01}^{\prime 2}}-\frac{i c^{2}}{f\left(c_{01}^{2}-i \omega c_{01}^{\prime 2}\right)}\left[1+\epsilon^{\prime}-\frac{c^{2}}{c_{01}^{2}-i \omega c_{01}^{\prime 2}}\right],
\end{gathered}
$$

where $f=\delta \omega / S \rho\left(c_{01}^{2}-i \omega c_{01}^{\prime 2}\right)$ is the reduced frequency.

Substituting (2.34) into (2.32), expanding the quantities $\beta_{1}, \beta_{2}$ in a series of " $f$," and neglecting the terms of the order $f^{1 / 2}$, we get

$$
\left[\left(x \mu-\frac{\rho c^{2}}{2}\right)-i \omega \mu^{\prime}\right]^{2}=\left(x \mu-i \omega \mu^{\prime}\right)^{2}\left(1-\frac{\rho c^{2}}{\mu-i \omega \mu^{\prime}}\right)^{1 / 2}\left(1-\frac{3 \rho c^{2}}{\left(1+\in^{\prime}\right)\left(9 \mu-8 i \omega \mu^{\prime}\right)}\right)^{1 / 2} .
$$

Considering a liquid medium, we write $\lambda=\mu=0$ and (2.35) simplifies to

$$
\left[\frac{H}{2}+\frac{\rho c^{2}}{2}+i \omega \mu^{\prime}\right]^{2}=\left(\frac{H}{2}+i \omega \mu^{\prime}\right)^{2}\left(1-\frac{i \rho c^{2}}{\omega \mu^{\prime}}\right)^{1 / 2}\left(1-\frac{3 i \rho c^{2}}{8\left(1+i \in_{0}\right) \omega \mu^{\prime}}\right)^{1 / 2},
$$

where $\epsilon_{0}=3 \gamma^{2} T_{0} / 8 \rho S \omega \mu^{\prime}$, which is deduced from (2.26).

Also, from (2.28), $x \mu=(1-H / 2 \mu) \mu=-H / 2$.

Rationalizing and squaring (2.36), we get

$$
\left(\frac{H}{2 \omega \mu^{\prime}}+\frac{\rho c^{2}}{2 \omega \mu^{\prime}}+i\right)^{4}=\left(\frac{H}{2 \omega \mu^{\prime}}+i\right)^{4}\left(1-\frac{i \rho c^{2}}{\omega \mu^{\prime}}\right)\left(1-\frac{3 \rho c^{2} \in_{0}}{8\left(1+\epsilon_{0}^{2}\right) \omega \mu^{\prime}}-\frac{3 i \rho c^{2}}{8\left(1+\in_{0}^{2}\right) \omega \mu^{\prime}}\right) \text {. }
$$

Expanding (2.37) and equating the real parts only, we get

$$
\begin{aligned}
\left(\varsigma_{0}+\frac{V_{0}^{2}}{2}\right)^{4}-6\left(\varsigma_{0}+\frac{V_{0}^{2}}{2}\right)^{2}+1= & \left(\varsigma_{0}^{4}-6 \varsigma_{0}^{2}+1\right)\left[1-\frac{3 V_{0}^{2} \epsilon_{0}}{8\left(1+\epsilon_{0}^{2}\right)}-\frac{3 V_{0}^{4}}{8\left(1+\epsilon_{0}^{2}\right)}\right] \\
& +4 V_{0}^{2} \varsigma_{0}\left(\varsigma_{0}^{2}-1\right)\left[1-\frac{3 V_{0}^{2} \epsilon_{0}}{8\left(1+\epsilon_{0}^{2}\right)}+\frac{3}{8\left(1+\epsilon_{0}^{2}\right)}\right]
\end{aligned}
$$

where $V_{0}^{2}=c^{2} / \omega \mu^{\prime} / \rho$ and $\varsigma_{0}=H / 2 \omega \mu^{\prime}$.

For different values of $\varsigma_{0}=H / 2 \omega \mu^{\prime}$, the values of $V_{0}$ are calculated for some specific value of $\in=\gamma^{2} T_{o} / S \rho\left[(\lambda+2 \mu)-i \omega\left(\lambda^{\prime}+2 \mu^{\prime}\right)\right]$ and the results are plotted in graphs. From these graphs, we find that the maximum value of the phase velocity $V_{0}$ of Rayleigh waves in viscoelastic liquid decreases as $\in$ changes from -0.1 to 0.1 .

When $\in=-0.1, V_{0}$ vanishes at $\varsigma_{0}=H / 2 \omega \mu^{\prime}=0$. It attains its maximum value 0.77850 at $\varsigma_{0}=0.1$, then decreases gradually as $\varsigma_{0}$ increases, and finally vanishes again at $\varsigma_{0}=0.5$ (Figure 2.2).

When $\in=0, V_{0}$ vanishes at $\varsigma_{0}=H / 2 \omega \mu^{\prime}=0$, attains a maximum value 0.43444 at $\varsigma_{0}=$ 0.2 , then gradually decreases as $\varsigma_{0}$ increases and vanishes again at $\varsigma_{0}=0.5$ (Figure 2.3). 
3892 Rayleigh waves in a viscoelastic half-space

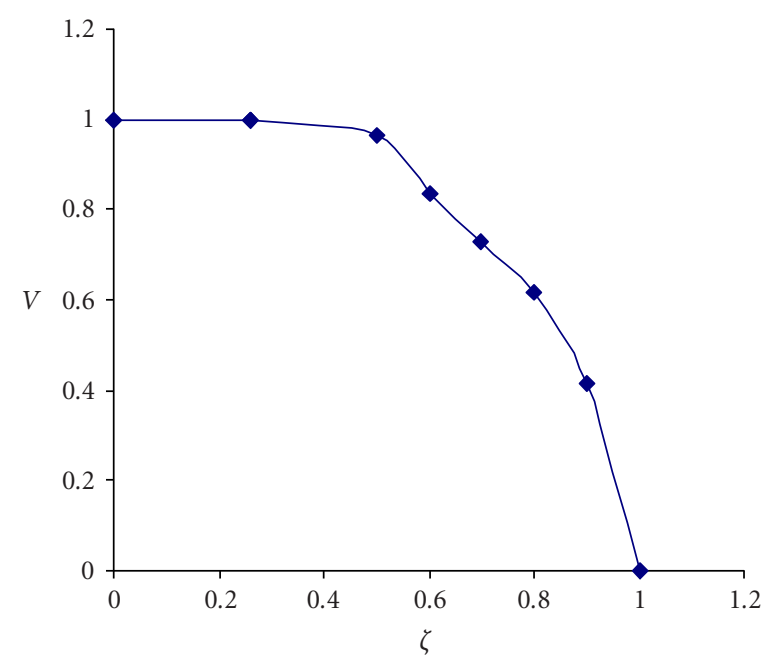

Figure 2.5. $\theta=1 / 2$.

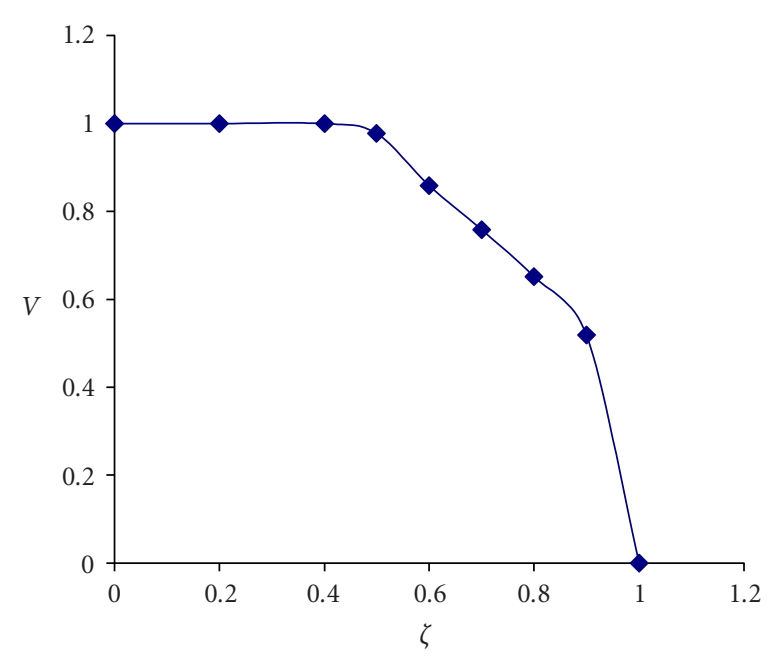

Figure 2.6. $\theta=1 / 3$.

When $\in=+0.1, V_{0}$ vanishes at $\varsigma_{0}=H / 2 \omega \mu^{\prime}=0$, attains a maximum value 0.34443 at $\varsigma_{0}=0.2$, then decreases gradually as $\varsigma_{0}$ increases, and vanishes again at $\varsigma_{0}=0.5$ (Figure 2.4).

In all the above three cases, the phase velocity $V_{0}$ ceases to exist at two particular values of $\varsigma_{0}$, which are $\varsigma_{0}=0$ and $\varsigma_{0}=0.5$, that is, when there is no initial stress and when the initial stress is equal to the product of the angular frequency $\omega$ of Rayleigh waves and the rigidity $\mu^{\prime}$ of the fluid. But the maximum values of $V_{0}$ are different for different values of $\in$. 


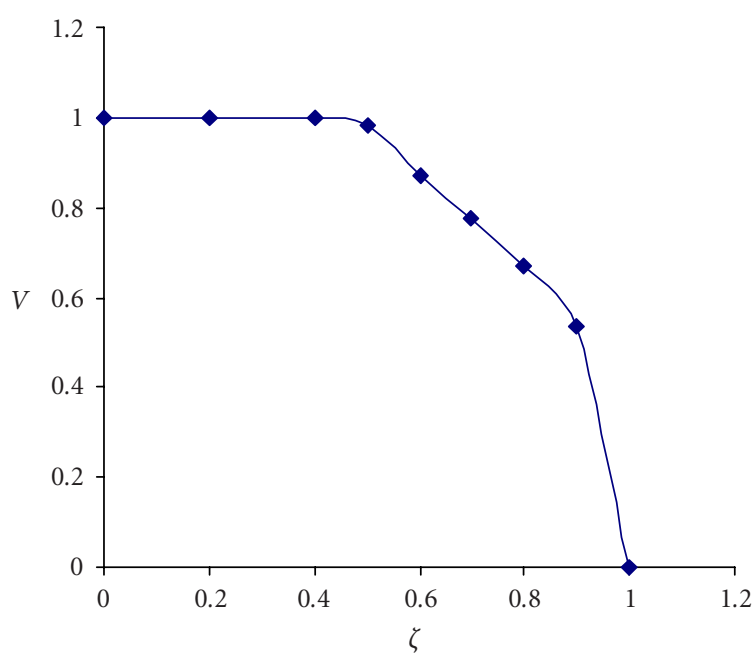

Figure 2.7. $\theta=1 / 4$. Variation of velocity of Rayleigh waves with initial stress when viscous effect is neglected (Figures 2.5, 2.6, 2.7).

If we neglect the viscous properties of the half-space, then $\mu^{\prime}=0$ and (2.35) reduces to

$$
\left[2(1-\varsigma)-V^{2}\right]^{2}=4(1-\varsigma)^{2}\left[1-V^{2}\right]^{1 / 2}\left[1-V^{2} \theta\right]^{1 / 2},
$$

where $\varsigma=H / 2 \mu, V^{2}=c^{2} / c_{2}^{2}$, and $\theta=c_{2}^{2} /(1+\in) c_{1}^{2}$.

Ignoring the initial stress, that is, with $H=0,(2.39)$ reduces to

$$
\left[2-V^{2}\right]^{2}=4\left[1-V^{2}\right]^{1 / 2}\left[1-V^{2} \theta\right]^{1 / 2} .
$$

This frequency equation for Rayleigh waves in an elastic solid medium is in perfect agreement with that obtained by Lockett [5]. Comparing (2.39) and (2.40), we find that in the result obtained by Lockett, the phase velocity of Rayleigh waves depends on the coupling coefficient $\theta=c_{2}^{2} /(1+\in) c_{1}^{2}$ between temperature and strain fields, but the results of the present authors show that the phase velocity depends on the initial stress also, besides the factor $\theta$ in an elastic solid medium.

If we vary $\varsigma$ keeping $\theta$ fixed, then the trend of phase velocity of Rayleigh waves changes remarkably, which is quite a new result in this paper. This fact may be used to understand the nature of Rayleigh waves accurately in seismology.

For different values of $\varsigma=H / 2 \mu$, the values of $V$ are calculated for some specific values of $\theta=c_{2}^{2} /(1+\in) c_{1}^{2}$ and these results are plotted in graphs. From these graphs, we find that the maximum and minimum values of phase velocity of Rayleigh waves are the same which are 1 and 0 , respectively, for $\zeta=0$ and 1 if we take $\theta=1 / 2,1 / 3$, or $1 / 4$ (Figures 2.5, 2.6, 2.7). Here, $\zeta=0$ implies that there is no initial stress and $\zeta=1$ implies that the initial hydrostatic stress is twice the rigidity of the solid elastic medium. We also note from the graphs that due to different values of $\theta$, there are slight variations in the overall 
nature of the graphs. This signifies that in an elastic solid medium, the phase velocity of Rayleigh waves depends much more on the initial stress of the medium than the coupling coefficient $\theta$ between temperature and strain fields. This finding is also of paramount importance in seismology.

\section{References}

[1] M. A. Biot, Mechanics of Incremental Deformations. Theory of Elasticity and Viscoelasticity of Initially Stressed Solids and Fluids, Including Thermodynamic Foundations and Applications to Finite Strain, John Wiley \& Sons, New York, 1965.

[2] P. Caloi, Comportement des ondes de Rayleigh dans un milieu firmo-élastique indéfini, Publ. Bureau Central Seismol. Internat. Sér. A. Trav. Sci. 17 (1950), 89-108.

[3] D. S. Chandrasekharaiah, One-dimensional wave propagation in the linear theory of thermoelasticity without energy dissipation, J. Thermal Stresses 19 (1996), 695-710.

[4] T. K. Das and P. R. Sengupta, Effect of gravity on visco-elastic surface waves in solids involving time rate of strain and stress of first order, Sadhana 17 (1992), part 2, 315-323.

[5] F. J. Lockett, Effect of thermal properties of a solid on the velocity of Rayleigh waves, J. Mech. Phys. Solids 7 (1958), no. 1, 71-75.

[6] W. Nowacki, Thermoelasticity, Addison-Wesley, London, 1962.

[7] W. Nowacki and M. Sokołowski, Propagation of thermoelastic waves in plates, Arch. Mech. (Arch. Mech. Stos.) 11 (1959), no. 6, 715-727.

Sushil Kumar Addy: Department of Mathematics, Jamshedpur Co-operative College, Jamshedpur Ranchi University, 831001 Jharkhand, India

E-mail address: skaddy@sify.com

Nil Ratan Chakraborty: Department of Physics, Jamshedpur Co-operative College, Jamshedpur Ranchi University, 831001 Jharkhand, India

E-mail address: nil_c53@yahoo.co.in 


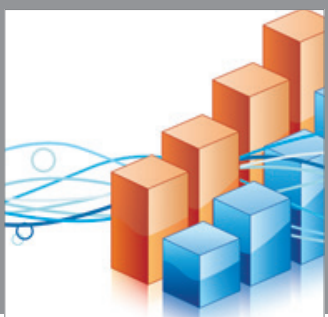

Advances in

Operations Research

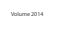

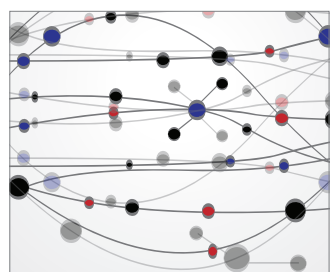

\section{The Scientific} World Journal
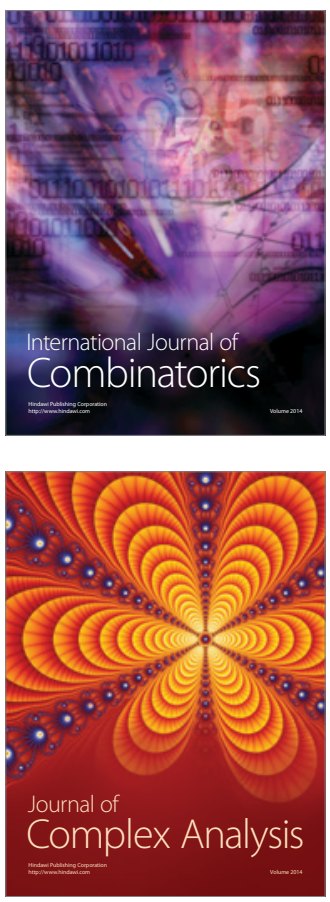

International Journal of

Mathematics and

Mathematical

Sciences
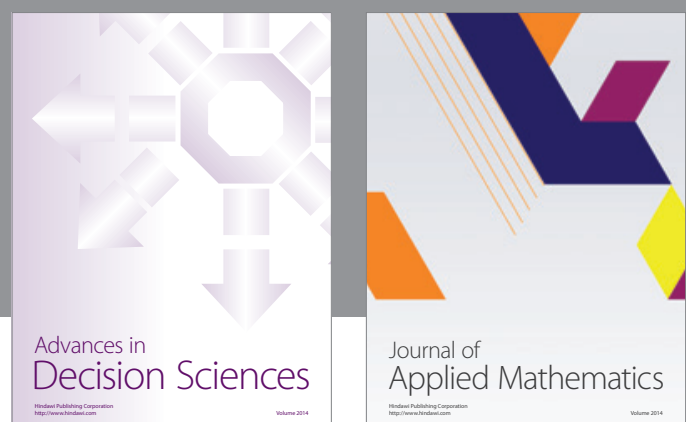

Journal of

Applied Mathematics
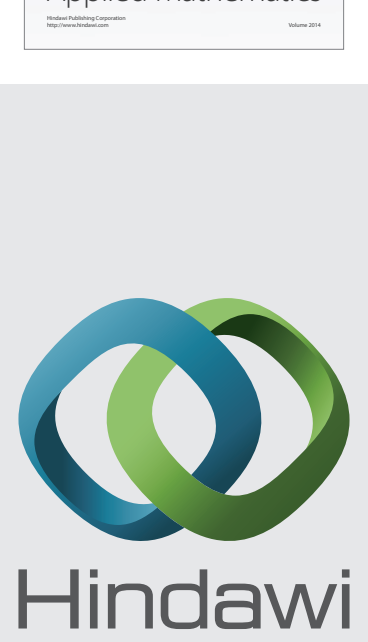

Submit your manuscripts at http://www.hindawi.com
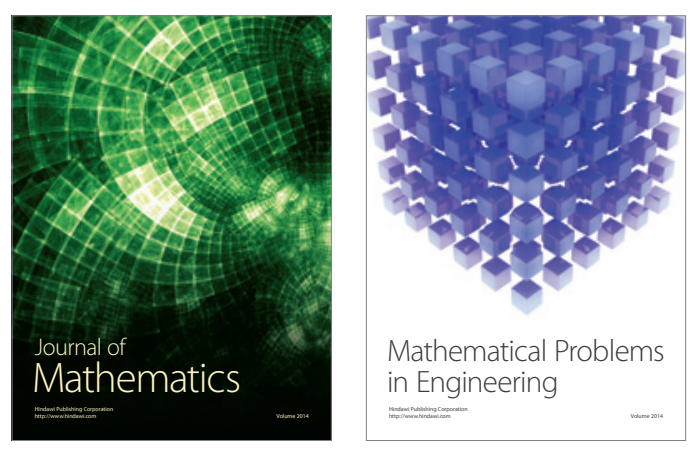

Mathematical Problems in Engineering
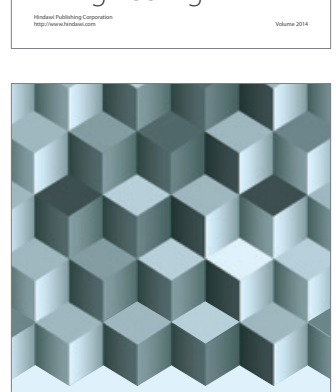

Journal of

Function Spaces
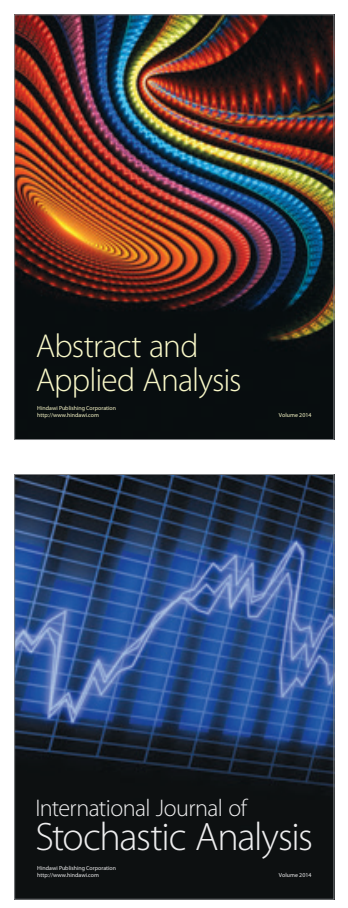

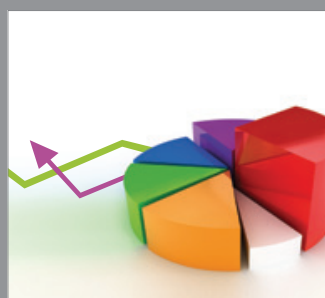

ournal of

Probability and Statistics

Promensencen
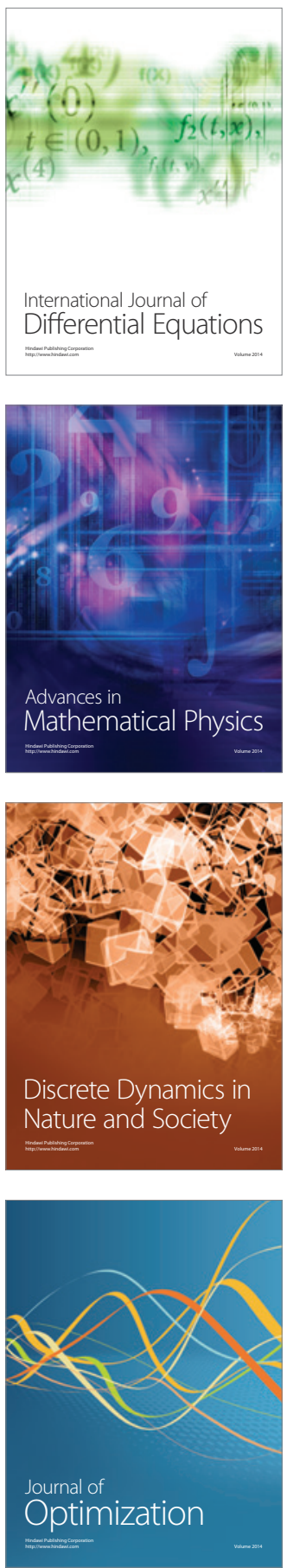